\title{
Molecular Structure of Water-Like Positronium Complexes
}

\author{
F. Rolim And J.R. MOHALlem* \\ Laboratório de Átomos e Moléculas Especiais \\ Departamento de Física, ICEx, Universidade Federal de Minas Gerais \\ P.O. Box 702, 30123-970, Belo Horizonte, MG, Brasil
}

We resort to a previously introduced molecular model for positron complexes to study the molecular structures of $\mathrm{Ps}_{2} \mathrm{O}$ and $\mathrm{PsOH}$ and to explore their analogies and differences from the molecular structure of water.

PACS numbers: 36.10.-k, 36.10.Dr, 31.30.-i, 82.30.Gg

\section{Introduction: the molecular model for positron complexes}

It is well known that positrons can live long enough to form complexes with atoms. The theoretical study of these complexes is important to interpret experimental data from both positron scattering by atoms and molecules and positron annihilation spectroscopy.

There are typically two different kinds of positron complexes. In one of them a positron attaches to a neutral or ionic atom or molecule A with a positive positron affinity, yielding the formation of the complex $\left[\mathrm{e}^{+} ; \mathrm{A}\right]$. In the other kind, a pair $\left(\mathrm{e}^{-}, \mathrm{e}^{+}\right)$(the positronium (Ps) atom) acts as a light isotope of hydrogen and binds to matter in almost the same way as the $\mathrm{H}$ atom does. Examples considered here are the two different species of positronic water, $[\mathrm{PsOH}]$ and $\left[\mathrm{Ps}_{2} \mathrm{O}\right]$.

Most theoretical studies of positron complexes consider the three different types of particles: heavy nuclei, light electrons and positrons in the same footing and aim to treat all the correlations between any pair of them. As a consequence, these calculations are computationally quite hard and limited to very small systems. We have found, on the other hand, that treating a positron as a light nucleus and performing an adiabatic separation of the electronic and nuclear motions, a lot of valuable information can be obtained from the potential energy surface (PES)

*corresponding author; e-mail: rachid@fisica.ufmg.br 
for nuclei and positron motions $[1,2]$. Despite the need of a large adiabatic correction to positron motion, everything else works as in standard molecular structure calculations. Of course we have to be careful in analyzing the outputs, when comparing with typical molecular calculations. For example, for a general complex $\left[\mathrm{e}^{+} ; \mathrm{A}\right]$ the point of minimum energy of the PES gives the geometry of the parent A system but just an approximation to the average position of the positron. Even though, predictions of positron bonding sites and geometry relaxation [2] and estimative of annihilation rates [1] are available within the molecular model.

In what follows we consider a complex containing just one positron, the extension to more positrons being trivial. We drop the notation for the nuclear coordinates $\boldsymbol{R}$, for simplicity, but the nuclear degrees of freedom are considered anyway in the Born-Oppenheimer (BO) approximation. The symbol A represents a neutral parent atomic or molecular system and $r$ stands for positron coordinates. Atomic units (a.u.) are used throughout.

The non-relativistic $N$-electron Hamiltonian, in which the kinetic energy terms of heavy particles are disregarded, is written as

$$
H=-\frac{\nabla_{r}^{2}}{2}-\sum_{i}^{N} \frac{\nabla_{i}^{2}}{2}+V,
$$

with conventional notation, $V$ representing the Coulomb potential energy of all particles.

Let us consider a general complex divided in two sub-systems. To keep the previous notation we call the positron and the $n_{B}$ electrons occupying orbitals $\phi_{B}$ as "sub-system $B$ ", so that "sub-system $A$ " is composed by the heavy nuclei and the remaining $n_{A}$ electrons occupying orbitals $\phi_{A}$. The procedure of considering momentum conservation just in sub-system $B$ lead to $\boldsymbol{\nabla}_{r}=-\sum_{i=1}^{n_{B}} \boldsymbol{\nabla}_{i}$. Due to the smallness of the positron mass, crossing (mass polarization) terms could be relevant but, since the positron can hardly attach more than one electron, they become negligible so that $\nabla_{r}^{2} \simeq-\sum_{i=1}^{n_{B}} \nabla_{i}^{2}$. This result will be taken into Eq. (1) to generate the model electronic Hamiltonian. This procedure would however distinguish electrons, so that we perform just a single sum over all $N$ electrons but impose no interaction of sub-systems $A$ and $B$ in calculations involving this term, a procedure that is symbolized by multiplying it by a $\delta_{A B}$, so that

$$
H=\sum_{i}^{N}\left(-\frac{\nabla_{i}^{2}}{2} \delta_{A B}-\frac{\nabla_{i}^{2}}{2}\right)+V .
$$

In practice, this means that matrix elements of the first operator between orbitals of different sub-systems are forced to vanish, that is, $\left\langle\phi_{A}\left|-\frac{\nabla_{i}^{2}}{2} \delta_{A B}\right| \phi_{B}\right\rangle=0$. 
In consequence, the Hamiltonian becomes a pure electronic operator and the positron is treated on a common footing with the other nuclei except that a large mass correction is introduced for its motion, as a consequence of the form (2) for the Hamiltonian. In the variational adiabatic approximation, the PES for nuclear motion is obtained by

$$
\epsilon(\boldsymbol{R}, r)=\frac{\langle\Phi|H| \Phi\rangle}{\langle\Phi|\mathbf{1}| \Phi\rangle}
$$

where $\Phi$ is a variational electronic wave function which depends parametrically on $\boldsymbol{R}$ and $r$ in the sense that $\Phi$ is normalizable for all values of these coordinates. As a matter of fact, for present applications, the parametric dependence of $\epsilon$ and $\Phi$ on the nuclear coordinates $\boldsymbol{R}$ is taken as in the usual BO approximation.

To solve this equation for general molecules, an electronic self-consistent-field-molecular-orbital-configuration-interaction (SCF-MO-CI) method has been implemented as a simple modification of the Gamess code [3, 4]. All the calculations reported here use this code.

\section{The molecular structure of $\left[\mathrm{Ps}_{2} \mathrm{O}\right]$ and $[\mathrm{PsOH}]$}

Complexes with two positrons seem unreliable in practical work, but are the interesting matter to theoretical study. The fact that the positronic water, $\mathrm{P}_{\mathrm{s}_{2}} \mathrm{O}$, could be bound was predicted by Jiang and Schrader [5] with the use of fully correlated diffusion Monte Carlo (DMC) method, yielding the total energy of -75.6270 a.u. and a binding energy of $1.67 \pm 0.08 \mathrm{eV}$ relative to the products $\mathrm{O}+$ Ps. They suggest the system to have a spherical symmetry with the two positrons occupying outher $1 s$ orbitals centered on the $\mathrm{O}$ atom (the atomic model). The stability of $[\mathrm{PsOH}]$ against dissociation of positron and positronium was predicted in Ref. [6], keeping frozen the geometries of the parent $\mathrm{OH}$ or $\mathrm{OH}^{-}$and with no other structure considerations. First tests with the molecular model show shallow minima of the PES against variation of bond distances (Ps-O being the more relevant) and the bond angle $\mathrm{Ps}_{\mathrm{s}}-\mathrm{O}-\mathrm{Ps}(\mathrm{H})$. To explore analogies with the structure of the water molecule we study first the barrier to linearity. We first perform a geometry optimization of each system. Then keeping constant the bond distance we vary the angle. Our results are displayed in Fig. 1 and the Table, and can be interpreted in terms of the molecular model. For the water molecule, it is well known that the localization of the bonds forces them to repel each other, including the lone pair, leading to its definite geometry and a large barrier to linearity. In the case of $\mathrm{Ps}_{2} \mathrm{O}$ no barrier has been found, see Fig. 1c. The explanation is that the two positrons (and thus the two Ps atoms) present a large deal of delocalization, so that bond repulsion takes no effect. On the other hand, the two Ps atoms must have their motion strongly correlated, occupying symmetric positions relative to the central $\mathrm{O}$ atom, in classical words. This explains the "linear configuration" in internal coordinates, advanced by the vanishing barrier, and a spherical picture of 
TABLE

Bond angles and barriers to linearity for water and water-like positronium complexes.

\begin{tabular}{l|c|c|c|l}
\hline \hline Complex & OH bond & OPs bond & Angle & Barrier \\
\hline $\mathrm{H}_{2} \mathrm{O}$ & 1.810 & - & 104.5 & 0.0556 \\
$\mathrm{PsOH}$ & 1.779 & 1.853 & 119.3 & 0.0089 \\
$\mathrm{Ps}_{2} \mathrm{O}$ & - & 1.810 & 180.0 & 0.00
\end{tabular}

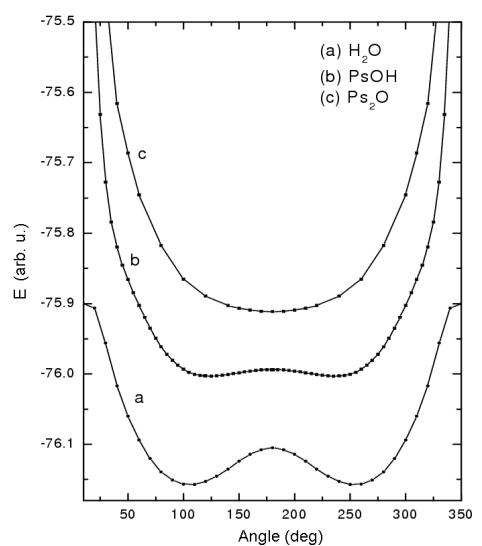

Fig. 1. $C_{2 v}$ cuts of the potential energy surfaces for water and water-like positronium complexes showing barriers to linearity.

(a)

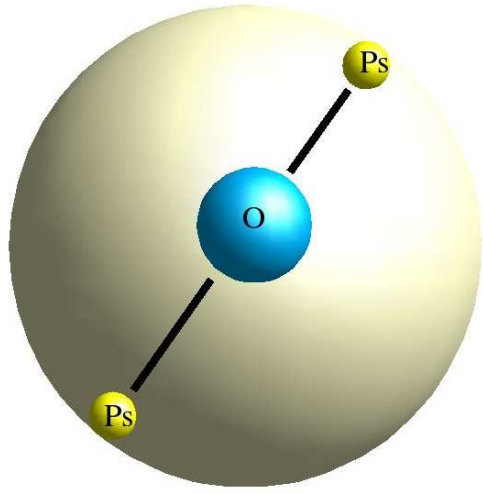

(b)

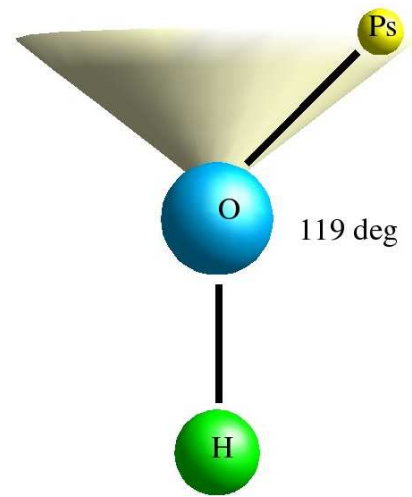

Fig. 2. Proposed molecular structures of (a) $\mathrm{Ps}_{2} \mathrm{O}$ and (b) $\mathrm{PsOH}$.

the system from outside, see Fig. 2a, which turns out to be in agreement with the spherical atomic model of Ref. [5].

The concept of molecular structure of Ps complexes becomes more meaningful in the case of PsOH. Geometry optimization leads to a bend structure with the bond angle of $119.3^{\circ}$, that is, a small but finite barrier to linearity appears, see 
Fig. 1b, and the $\mathrm{O}-\mathrm{H}$ bond distance is somewhat reduced as well. The two heavy nuclei define a reference axis, which prevents spherical symmetry of Ps motion, leading to the cone model of Fig. $2 \mathrm{~b}$ as our picture for this system. We found this to be a general rule, that is, as the parent molecule becomes larger, a single positron tends to be localized in some molecular site [2, 7], instead of occupying an outer molecular orbital.

The minimum of the PES of $\mathrm{PsOH}$ is -76.0175 a.u. Comparing with the DMC value of -75.9815 a.u. [6] we note that the difference is very small, about $10 \%$ of the ground state Ps energy, -0.25 a.u. This feature is unexpected, and could be related to the fact that in the calculation of the $\mathrm{O}-\mathrm{H}$ bond is kept frozen [6]. Anyway, a general property is that the experimental result (or exact calculations) will be an upper bound to our minimum point energy. In the same way, the minimum point energy of the corresponding common molecule $\left(\mathrm{H}_{2} \mathrm{O}\right.$ in this case) will be a lower bound. This property can be explored in the study of larger complexes.

Finally, in Ref. [1], we proposed that the pair annihilation rate of Ps, $\Gamma=2.0 \times 10^{9} \mathrm{~s}^{-1}$, is a lower bound to the annihilation rate per positron in any complex in a state that breaks in one or more Ps atoms. Thus for $\mathrm{Ps}_{2} \mathrm{O}$ we predict $\Gamma \gtrsim 4.0 \times 10^{9} \mathrm{~s}^{-1}$ which is in consonance with the calculation of Ref. [5]. In the case of $\mathrm{PsOH}$, any state supported by the ground state PES will dissociate in $\mathrm{Ps}+\mathrm{OH}$, as discussed in Ref. [8]. We have really found it by stretching the coordinate $\mathrm{Ps}_{\mathrm{s}} \mathrm{OH}$, allowing the bond $\mathrm{OH}$ to relax. We can thus propose for the annihilation rate of such a state the value $\Gamma \gtrsim 2.0 \times 10^{9} \mathrm{~s}^{-1}$.

\section{Further remarks}

We have discussed the application of the molecular model for positron complexes to $\left[\mathrm{Ps}_{2} \mathrm{O}\right]$ and $[\mathrm{PsOH}]$. The molecular structure that comes out, when we take the positron as a light nucleus, permits us to get a "chemical" picture of the complexes that can be further explored in the predictions of their properties.

We acknowledge Capes and $\mathrm{CNPq}$ for supporting this work.

\section{References}

[1] J.R. Mohallem, F. Rolim, C.P. Gonçalves, J Phys. B, At. Mol. Opt. Phys. 37, 1045 (2004).

[2] J.R. Mohallem, C.P. Gonçalves, J. Chem. Phys. 121, 5553 (2004).

[3] C.P. Gonçalves, J.R. Mohallem, Theor. Chem. Acc. 110, 367 (2003).

[4] C.P. Gonçalves, J.R. Mohallem, J. Comput. Chem. 25, 1736 (2004).

[5] N. Jiang, D.M. Schrader, Phys. Rev. Lett. 81, 5113 (1998).

[6] D. Bressanini, M. Mella, G. Morosi, J Chem. Phys. 109, 5931 (1998).

[7] M. Tachikawa, R.J. Buenker, M. Kimura, J. Chem. Phys. 119, 5005 (2003).

[8] D.M. Schrader, J. Chem. Phys. 104, 3147 (1996). 